\title{
LOS DERECHOS FUNDAMENTALES EN AMÉRICA LATINA: UNA PERSPECTIVA NEOCONSTITUCIONALISTA
}

\author{
Miguel Carbonell Sánchez* \\ Universidad Nacional Autónoma de México, México \\ miguel@miguelcarbonell.com
}

\begin{abstract}
RESUMEN: El autor analiza la evolución que han tenido los derechos fundamentales en Latinoamérica en las últimas décadas. En primer lugar desde un punto de vista normativo, el autor identifica, por una parte, el proceso de universalización que se ha producido respecto de los derechos fundamentales -en cuanto a su consagración jurídica y su alcance geográfico-, y un proceso de especificación de los mismos - respecto de sus destinatarios y de su objeto-, por otra. En segundo lugar, desde un punto de vista teórico, el autor analiza la evolución que ha experimentado la doctrina relativa a los Derechos Fundamentales en las últimas décadas, y el influjo del neoconstitucionalismo, el constitucionalismo norteamericano y europeo, como teorías clave para el actual desarrollo de los mismos. Finalmente, el autor identifica los principales riesgos para el desarrollo y consagración de los derechos fundamentales en Latinoamérica, como serían el discurso de la seguridad, el retorno al autoritarismo y la precaria cultura jurídica en la materia.
\end{abstract}

Palabras clave: derechos fundamentales, especificación, universalización, neoconstitucionalismo.

ABSTRACT: The author analyses the evolution that have taken Fundamental Rights in Latin America through the last decades. In the first place, from a normative perspective, the author identifies on one hand, the universalization progress that has occurred regarding the Fundamental Rights -in relation with its legal consecration and geographic scope- and a specification process -concerning its recipient and subject- on the other.

* Doctor en Derecho por la Universidad Complutense de Madrid, Espańa. Licenciado en Derecho por la Facultad de Derecho de la UNAM. Esta ponencia fue presentada el ańo 2009 en el X Congreso Iberoamericano de Derecho Constitucional celebrado en Lima en el mes de septiembre, además de ser presentada el año 2010 en el II Congreso de Derechos Humanos, del que trata ese número. 
In the second place, from a theoretical standpoint, the author analyze the development experienced by the doctrine on Fundamental Rights in recent decades and the influence of neoconstitutionalism, American and European constitutionalism, as key theories for the its present progress. Finally, the author identifies the main risks for the development and consecration of Fundamental Rights in Latin America, as would be the discourse of security, the return to authoritarianism and the precarious legal culture on the field.

Keywords: fundamental rights, specification, universalization, neoconstitutionalism.

\section{INTRODUCCIÓN}

Los derechos fundamentales en América Latina han sufrido un proceso de intensa evolución en los últimos 30 ańos. Si nos remontamos a la segunda mitad de la década de los 70 del siglo pasado, veremos las grandes diferencias que existen respecto a lo que tenemos hoy en día en materia de derechos. Lo que en otras latitudes tomó dos siglos, en América Latina se ha conseguido, con muchos problemas, defectos y riesgos, en menos de tres décadas.

Desde luego, un análisis responsable debería ser capaz de distinguir al menos tres planos de análisis y observar el desarrollo que se ha producido en cada uno de ellos:

1) Un plano normativo, en el que se tuvieran en cuenta los textos constitucionales y los catálogos de derechos previstos en los tratados internacionales, tanto de carácter universal como de alcance regional.

2) Un plano teórico, a fin de dar cuenta de la evolución y crecimiento del "discurso" científico sobre los derechos.

3) Un plano sociológico, con el objetivo de poner en evidencia la manera en que los derechos se han ido aplicando, con enormes dificultades e incluso con preocupantes retrocesos, en la práctica constitucional de América Latina.

En las páginas que siguen haremos referencia solamente a los dos primeros niveles, aunque en el último apartado haremos un somero análisis de algunos riesgos nada teóricos para los derechos fundamentales.

\section{PLANO NORMATIVO}

Desde el primer nivel de análisis se puede observar un proceso siempre creciente y (hasta el día de hoy) en términos generales imparable de expansión de los derechos enunciados por las constituciones de la región. Este proceso está presente a todo lo largo de la histo- 
ria de los derechos, comenzando por sus "cunas originales", que fueron Francia y los Estados Unidos. La expansión de los catálogos de derechos se ha producido por un doble fenómeno de universalización de los titulares de los derechos (como efecto de la aceptación ampliamente compartida de la universalidad de los derechos) y de la mayor especificación que van adquiriendo sus contenidos.

La universalidad es una característica que ha acompañado a los derechos fundamentales desde su nacimiento. De hecho, uno de los primeros catálogos de derechos -que además es, quizá, el más importante de todos los tiempos- ya contenía la aspiración a la validez universal de los derechos; en efecto, la lectura de los artículos que integran la Declaración de los Derechos del Hombre y del Ciudadano, de agosto de 1789 (conocida simplemente como Declaración francesa), pone en evidencia que sus normas están dirigidas no solamente a los franceses, sino a todas las personas y que su ámbito de validez racional no se limitaba a Francia, sino que pretendía ir más allá de sus fronteras, como efectivamente lo hizo.

Como lo explica García Manrique, la universalidad de la Declaración francesa tiene dos sentidos: uno textual y otro funcional; la universalidad textual se desprende de la forma en que están redactados sus preceptos, que no se dirigen solamente a los franceses, sino a todos los hombres; la universalidad funcional se proclama en virtud de que la Declaración expresa ideas que pretenden alcanzar una validez universal e intenta recoger los principios que deben servir para estructurar no solamente a la sociedad francesa, sino a cualquier sociedad:

"La Declaración puede considerarse universal en dos sentidos: textualmente y funcionalmente... En primer lugar, la Declaración es textualmente universal como efecto necesario del modelo iusnaturalista que inspira su forma y su contenido... expresa ideas que pretenden una validez universal... la Declaración es funcionalmente universal porque ha servido como referente ideológico en todo el mundo a lo largo de dos siglos. Ello se debe, por supuesto, al lenguaje universal de su texto, pero no solo a él, sino también a dos circunstancias no imputables a la voluntad de sus redactores: una es que la Declaración refleja la filosofía moral, política y jurídica de la modernidad... y que ha alimentado de una u otra forma la mayor parte de los proyectos políticos de los siglos XIX y XX, hasta nuestros días: la otra es que la situación social y política europea de finales del siglo XVIII era lo bastante similar a la francesa como para que la Declaración tuviera impacto inmediato, profundo y duradero más allá de las fronteras de Francia"1.

García Manrique, Ricardo. "Sentido y contenido de la Declaración de 1789 y textos posteriores”, Historia de los derechos fundamentales, tomo II, volumen III. Madrid, España: Dykinson, Universidad Carlos III, 2001, pp. 247-248. 
La universalidad, además, es una consecuencia de la fuerte influencia iusnaturalista del primer constitucionalismo: si los derechos que entonces se enunciaban eran "naturales", es obvio que tenían que ser reconocidos por igual a todas las personas, puesto que todas ellas comparten la misma "naturaleza"; en palabras de Stephane Rials, "si existe un orden natural racional cognoscible con evidencia, sería inconcebible que fuera consagrado con variantes significativas según las latitudes" 2 .

En términos generales la universalidad de los derechos fundamentales puede ser estudiada desde dos distintos puntos de vistas. Desde el punto de vista de la teoría del derecho la universalidad tiene que ver con la forma en que están redactados los preceptos que contienen derechos. Si su forma de redacción permite concluir que un cierto derecho se adscribe universalmente a todos los sujetos de una determinada clase (menores, trabajadores, campesinos, ciudadanos, mujeres, indígenas: lo importante es que esté adscrito a todas las personas que tengan la calidad establecida por la norma), entonces estamos ante un derecho fundamental universal.

Algunos autores han incluido la característica de la universalidad como requisito esencial para la identificación de los derechos fundamentales. Es decir, para ellos solamente serían fundamentales los derechos que sean universales. Es el caso de Luigi Ferrajoli, quien distingue entre los derechos fundamentales (asignados universalmente a todos los sujetos de una determinada clase) y los derechos patrimoniales (asignados a una persona con exclusión de los demás); así por ejemplo, la libertad de expresión, al ser reconocida constitucionalmente como un derecho de toda persona, sería un derecho fundamental; mientras que el derecho patrimonial sobre mi coche (derecho que comprende la posibilidad de usarlo, venderlo, agotarlo y destruirlo) excluye de su titularidad a cualquier otra persona ${ }^{3}$. En palabras del autor ${ }^{4}$ :

"Los derechos fundamentales -tanto los derechos de libertad como el derecho a la vida, y los derechos civiles, incluidos los de adquirir y disponer de los bienes objeto de propiedad, del mismo modo que los derechos políticos y los derechos sociales- son derechos 'universales' (omnium), en el sentido lógico de la cuantificación universal de la clase de sujetos que son sus titulares; mientras los derechos patrimoniales - del derecho de propiedad a los demás derechos reales y también los derechos de créditoson derechos singulares (singuli), en el sentido asimismo lógico de que para cada uno de ellos existe un titular determinado (o varios cotitulares, como en la copropiedad) con exclusión de todos los demás... Unos son inclusivos y forman la base de la igual-

2 Citado por García Manrique, Ricardo. "Sentido y contenido de la Declaración de 1789 y textos posteriores", cit., pp. 247-248.

3 Ferrajoli, Luigi. Derechos y garantías. La ley del más débil-4a edición-Madrid, España: Trotta, 2004, pp. 45 y ss.

$4 \quad$ Ibid., p. 46. 
dad jurídica... Los otros son exclusivos, es decir, excludendi alios, y por ello están en la base de la desigualdad jurídica”.

Siguiendo desde la misma perspectiva de teoría del derecho hay que distinguir, como lo ha explicado Robert Alexy, entre la universalidad con respecto a los titulares y la universalidad respecto a los destinatarios (obligados) de los derechos 5 . La primera consiste "en que los derechos humanos son derechos que corresponden a todos los seres humanos", con independencia de un título adquisitivo ${ }^{6}$. Los destinatarios (en cuanto que obligados por los derechos) serían no solamente los seres humanos en lo individual, sino también los grupos y los Estados. En este último caso, de acuerdo con Alexy, hay que diferenciar los derechos humanos absolutos de los derechos humanos relativos; los primeros son los que se pueden oponer frente a todos los seres humanos, a todos los grupos y a todos los Estados, mientras que los segundos -los relativos- solamente son oponibles a, por lo menos, un ser humano, un grupo o un Estado.

Alexy pone como ejemplo de derechos humanos absolutos el derecho a la vida, que debe respetarse por todos; un ejemplo de derecho humano relativo frente al Estado sería el derecho al voto, el cual debe ser respetado por el Estado del cual el individuo forma parte; un ejemplo de derecho humano relativo frente a un grupo sería el derecho de los niños a que sus familias les proporcionen asistencia y educación.

Aparte de la perspectiva de teoría del derecho, que es la que se acaba de explicar de forma muy resumida, la universalidad de los derechos debe también ser contemplada desde una óptica política, a partir de la cual dicha característica supondría la idea de que todos los habitantes del planeta, con independencia del país en el que hayan nacido y del lugar del globo en el que se encuentren, deberían tener al menos el mismo núcleo básico de derechos fundamentales, los cuales además tendrían que ser respetados por todos los gobiernos.

Desde luego, la forma en que ese núcleo básico podría plasmarse en los distintos ordenamientos jurídicos no tiene que ser uniforme para estar de acuerdo con los principios de justicia; la historia, cultura y pensamiento de cada pueblo o comunidad puede agregar, y de hecho históricamente ha agregado, una multiplicidad de matices y diferencias al conjunto de derechos fundamentales que establece su respectiva Constitución. En palabras de Konrad Hesse, “... la validez universal de los derechos fundamentales no supone uniformidad... el

5 Alexy, Robert. "La institucionalización de los derechos humanos en el Estado constitucional democrático", Derechos y libertades, número 8. Madrid, enero-junio de 2000, pp. 24-26.

6 Alexy no acepta que puedan haber derechos de grupo, es decir, derechos que no sean asignados a cada uno de los seres humanos en lo individual, si bien reconoce que pueden existir "derechos de comunidades" o "de Estados" (derechos de tercera generación, derecho al desarrollo); tales derechos, sin embargo, no serían derechos humanos, con lo cual -reconoce el autor-se perdería la carga valorativo-positiva que tiene el término, pero se obtendría la ventaja "de aguzar la vista para que estos derechos no devengan en derechos de funcionarios", "La institucionalización de los derechos humanos en el Estado constitucional democrático", cit., p. 25. 
contenido concreto y la significación de los derechos fundamentales para un Estado dependen de numerosos factores extrajurídicos, especialmente de la idiosincrasia, de la cultura y de la historia de los pueblos"7.

En la historia de los derechos hemos encontrados muchas formas distintas de redactarlos, si bien también se han dado muchas influencias entre los distintos textos que los recogen; influencias ideológicas, desde luego, pero también influencias "textuales", por medio de las cuales los poderes constituyentes han redactado de forma muy parecida las normas que establecen ciertos derechos.

La caracterización de los derechos fundamentales como derechos universales no solamente sirve para extenderlos sin distinción a todos los seres humanos y a todos los rincones del planeta, sino que también es útil para deducir su inalienabilidad y su no negociabilidad; en palabras del propio Ferrajoli, si tales derechos "son normativamente de 'todos' (los miembros de una determinada clase de sujetos), no son alienables o negociables, sino que corresponden, por decirlo de algún modo, a prerrogativas no contingentes e inalterables de sus titulares y a otros tantos límites y vínculos insalvables para todos los poderes, tanto públicos como privados" 8 .

Que no sean alienables o negociables significa, en otras palabras, que los derechos fundamentales no son disposibles. Su no disponibilidad es tanto activa (puesto que no son disponibles por el sujeto que es su titular), como pasiva (puesto no son disponibles, expropiables o puestos a disposición de otros sujetos, incluyendo sobre todo al Estado) ${ }^{9}$.

En este contexto, se puede afirmar que los derechos fundamentales, tomando en cuenta tanto su universalidad como su protección constitucional, se sitúan fuera del mercado y de los alcances de la política ordinaria. Esto significa que no puede existir una justificación colectiva que derrote la exigencia que se puede derivar de un derecho fundamental.

Tal como lo expresa Ronald Dworkin, "Los derechos individuales son triunfos políticos en manos de los individuos. Los individuos tienen derechos cuando, por alguna razón, una meta colectiva no es justificación suficiente para negarles lo que, en cuanto individuos, desean tener o hacer, o cuando no justifica suficientemente que se les imponga una pérdida o un perjuicio"10; en el mismo sentido, Robert Alexy seńala que "El sentido de los derechos fundamentales consiste justamente en no dejar en manos de la mayoría parlamentaria la decisión sobre determinadas posiciones del individuo, es decir, en delimitar el campo de

\footnotetext{
Hesse, Konrad, "Significado de los derechos fundamentales", en Benda et al. Manual de derecho constitucional. Madrid, España: IVAP-Marcial Pons, 1996, p. 85.

8 Ferrajoli, Luigi, op. cit. (n. 2), p. 39.

9 Ibid., p. 47.

10 Dworkin, Ronald. Los derechos en serio. Barcelona, España: Planeta-Agostini, 1993, p. 37.
} 
decisión de aquella..." ${ }^{11}$. Esto significa que frente a un derecho fundamental no pueden oponerse conceptos como el de "bien común", "seguridad nacional”, "interés público", "moral ciudadana”, etcétera. Ninguno de esos conceptos tiene la entidad suficiente para derrotar argumentativamente a un derecho fundamental.

En todas las situaciones en las que se pretenda enfrentar a un derecho fundamental con alguno de ellos el derecho tiene inexorablemente que vencer, si en verdad se trata de un derecho fundamental.

Ni siquiera el consenso unánime de los integrantes de una comunidad puede servir como instrumento de legitimación para violar un derecho fundamental, pues como señala Ferrajoli,

"Ni siquiera por unanimidad puede un pueblo decidir (o consentir que se decida) que un hombre muera o sea privado sin culpa de su libertad, que piense o escriba, o no piense o no escriba, de determinada manera, que no se reúna o no se asocie con otros, que se case o no se case con cierta persona o permanezca indisolublemente ligado a ella, que tenga o no tenga hijos, que haga o no haga tal trabajo u otras cosas por el estilo. La garantía de estos derechos vitales es la condición indispensable de la convivencia pacífica. Por ello, su lesión por parte del Estado justifica no simplemente la crítica o el disenso, como para las cuestiones no vitales en las que vale la regla de la mayoría, sino la resistencia a la opresión hasta la guerra civil" ${ }^{\prime 2}$.

La base normativa de la universalidad de los derechos humanos se encuentra, además de lo ya dicho, en los diversos pactos, tratados y convenciones internacionales que existen sobre la materia. El punto de partida de todas esas disposiciones -en sentido conceptual, no temporal, desde luego- se encuentra en la Declaración Universal de los Derechos del Hombre de 1948. Dicha Declaración, junto con la Carta de la ONU, supone el embrión de un verdadero "constitucionalismo global"13.

Norberto Bobbio sostiene que con la Declaración de 1948 se inicia una fase importante en la evolución de los derechos: la de su universalización y positivación, haciéndolos pasar de "derechos de los ciudadanos" a verdaderos derechos de (todos) "los hombres", o al menos "derechos del ciudadano de esa ciudad que no conoce fronteras, porque comprende a toda la humanidad"14.

11 Alexy, Robert. Teoría de los derechos fundamentales. Madrid, España: CEPC, 2002 (reimpresión), p. 412.

12 Ferrajoli, Luigi. Derecho y razón, p. 859.

13 Ferrajoli, Luigi. "Más allá de la soberanía y la ciudadanía: un constitucionalismo global”. En: Carbonell, Miguel (compilador). Teoría de la Constitución. Ensayos escogidos -4a edición- México: IIJ-UNAM, Porrúa, 2008, pp. 397 y ss.

14 Bоввіо, Norberto. L'eta dei diritti, pp. 23-24. 
Tiene razón, pero solamente en cuanto que la Declaración de 1948 supone un producto acabado de la vocación universal de los derechos; en realidad la característica de la universalidad ya estaba presente, como lo explicamos con anterioridad, desde las primeras cartas de derechos.

Lo importante en este punto es que, a partir de la Declaración de 1948, los derechos dejan de ser una cuestión interna de la incumbencia exclusiva de los Estados y saltan por completo al terreno del derecho y las relaciones internacionales. Los particulares se convierten en sujetos de ese nuevo derecho, antes reservado solamente a la actuación de los Estados y no de los individuos, en la medida en que tienen asegurado un estatus jurídico supranacional; incluso, bajo ciertas circunstancias, pueden acceder a una jurisdicción internacional para el caso de que consideren violados sus derechos. Los tribunales nacionales empiezan a aplicar las normas jurídicas internacionales y los problemas antes considerados como exclusivamente domésticos adquieren relevancia internacional; podemos afirmar, en consecuencia, que también en materia de derechos humanos -como en tantos otros aspectos- vivimos en la era de la interdependencia. Más adelante volveremos sobre este tema, en referencia específica a la creciente "internacionalización” de los derechos en América Latina.

Resumiendo lo que se ha dicho podemos afirmar que la universalidad de los derechos es un rasgo que puede ser analizado desde una doble perspectiva: desde el punto de vista en que está redactada una norma jurídica (lo que nos sirve para identificar a los derechos fundamentales, según el punto de vista de Ferrajoli, diferenciándolos de los derechos patrimoniales), y desde el punto de vista de su alcance geográfico (lo que nos indica que no serán universales aquellos derechos que se queden dentro de las fronteras de los Estados o estén asignados solamente -excludendi alios- a sus habitantes). Si lo primero tiene un significado estrictamente normativo, lo segundo encierra un enorme debate político y social, quizá uno de los más importantes de nuestro tiempo.

Por su parte, el proceso de especificación de los derechos, como lo explica Norberto Bobbio, se ha dado en virtud del paso del hombre abstracto al hombre en concreto, del individuo considerado solamente como ciudadano al individuo considerado en los distintos roles o estatus que puede tener en la sociedad -aunque no se trata de roles solamente sociales, sino también biológicos-. Para ese efecto se han tomado en cuenta diversos criterios de diferenciación: el sexo, la edad, las condiciones físicas, etcétera, que merecen un especial tratamiento y protección ${ }^{15}$.

A partir de las primeras décadas del siglo XX se empieza en consecuencia a hablar de derechos de los trabajadores, de los campesinos, de los niños, de los ancianos, de las personas con discapacidad, de los enfermos, de las mujeres, de los indígenas, de los consumido-

15 Ibid., pp. 62 y 68 , entre otras. 
res, de los inmigrantes, de las minorías sexuales, entre otros. La Constitución de Querétaro, de 1917, por ejemplo, realizó un verdadero salto en el tiempo al constitucionalizar derechos para grupos en situación de vulnerabilidad, como los campesinos y los trabajadores.

Actualmente, se habla incluso de los derechos de las generaciones futuras, que se verían amenazados por los posibles efectos de una guerra nuclear o de la devastación ecológica producida por la destrucción que sobre el medio ambiente tienen algunos procesos productivos. Como apunta Giancarlo Rolla, "En el constitucionalismo contemporáneo el hombre y la mujer son considerados en su calidad de personas históricamente determinadas, inmersos en la sociedad, personas concretas, consideradas en su existencia histórica y material, portadoras de múltiples necesidades y expectativas" ${ }^{16}$.

Si la universalización puso en claro que los derechos pertenecen a todas las personas, con independencia de su lugar de nacimiento o residencia, de sus características físicas, de sus preferencias y sus ideologías, la especificación quiere responder a las preguntas “¿qué hombre?” y “¿qué ciudadano?”17.

La especificación se ha producido sobre todo en el campo de los derechos sociales, en la medida en que la igualdad y la libertad genéricamente expresadas no han sido suficientes para proteger todos los intereses de grandes grupos humanos marginados o que conviven en la sociedad de forma desventajosa.

La especificación se refiere tanto a los destinatarios -es decir, a los sujetos- de los derechos, como a los bienes que protegen los derechos -es decir, al objeto de los mismos-; en consecuencia, de la misma forma en que hoy ya no se habla de ciudadano o de persona sin más, tampoco se regula la libertad o la igualdad sin más, sino que se ha avanzado en un proceso de determinación también de esos bienes, que se han ido haciendo cada vez más precisos.

En nuestra región, un primer momento especialmente importante dentro de los procesos que estamos analizando se observa ya con la Constitución guatemalteca, de 1985, la que no solamente nos ofrece un largo catálogo de derechos, sino que también se ocupa de clasificarlos, ofreciendo al lector una primera clave de lectura de gran relevancia (una cuestión que merece ser discutida aparte es si esa clasificación resulta más o menos acertada, más

16 Rolla, Giancarlo. "La actual problemática de los derechos fundamentales", en: Asamblea, número 3, Madrid, junio de 2000, p. 49. El mismo autor explica que "la tendencia a la especificación representa no tanto una ampliación de las posiciones subjetivas abstractamente tutelables, sino una técnica de codificación específica dirigida a concretar históricamente la materia de los derechos de la persona reconocidos en un determinado ordenamiento. Tal técnica se propone -quizá de forma optimista- codificar un catálogo de derechos de la persona sin lagunas, en contraposición a otros ordenamientos constitucionales propensos a recabar la tutela de nuevas posiciones subjetivas por vía jurisprudencial e interpretativa, sobre la base de formulaciones constitucionales esenciales", Rolla, Giancarlo. Derechos fundamentales, Estado democrático y justicia constitucional. México: UNAM, 2002, pp. 35-36.

17 Воввіо, Norberto, op. cit. (n. 14), p. 62. 
o menos exhaustiva y más o menos comprensible). Pero son sobre todo las constituciones de Brasil, en 1988, y de Colombia, en 1991, las que, con base en las experiencias europeas de la Segunda Posguerra, expanden hasta niveles previamente desconocidos los catálogos de derechos.

En Brasil el afán clasificatorio de otros documentos no preocupa demasiado a los autores de su texto constitucional, quienes decidieron redactar un extenso artículo 5 que cuenta con 78 fracciones, muchas de las cuales se dividen en distintos apartados; en este artículo se encuentran tanto cuestiones sustantivas como procedimentales (es decir, tanto derechos como garantías). La misma Constitución brasileña pone aparte los derechos sociales (artículos 6 a 11), el régimen jurídico de la nacionalidad (artículos 12 y 13) y los derechos políticos (artículos 14 a 16).

Una visión más moderna, incluso terminológicamente, se observa en el texto colombiano, de 1991, cuyo Título II se denomina “De los derechos, garantías y deberes". En ese Título se encuentra una clasificación que separa los derechos fundamentales, los derechos económicos sociales y culturales, los derechos colectivos y del ambiente, y los deberes y obligaciones (una distinción parecida puede observarse en la Constitución peruana de 1993). Es probable que la distinción entre derechos fundamentales y otro tipo de derechos (como los sociales) provenga de la no muy acertada fórmula del artículo 53 de la Constitución española, que parece distinguir los derechos "plenos", objeto de protección por todos los tribunales o mediante amparo ante el Tribunal Constitucional, de los simples principios que sirven para "informar" la legislación positiva, la práctica judicial y la actuación de los poderes públicos ${ }^{18}$.

La Constitución de Argentina, reformada en profundidad en 1994, contiene un capítulo segundo dedicado a "Nuevos derechos y garantías", dentro del cual se encuentran interesantes desarrollos para su catálogo de derechos, incluyendo medios de tutela oponibles a particulares (como el juicio de amparo, según el artículo 43 párrafo primero). Infortunadamente, se trata de un capítulo poco sistemático, ya que mezcla cuestiones no muy homogéneas, lo que probablemente no aporte una adecuada guía de lectura para quienes quieran profundizar en su conocimiento.

La Constitución mexicana ha ido incrementando paulatinamente el espacio dedicado a la regulación de los derechos fundamentales, si bien es cierto que lo ha hecho con escasa técnica legislativa y sin ningún prurito por salvaguardar una mínima sistemática ${ }^{19}$.

18 Un análisis de los alcances de dicho precepto puede verse en Jiménez CAMpo, Javier. Derechos fundamentales: concepto y garantías. Madrid, Espańa: Trotta, 1999.

19 Un estudio de tales derechos puede verse en Carbonell, Miguel. Los derechos fundamentales en México -3a edición-México: Porrúa, UNAM, CNDH, 2009. Las críticas a su falta de sistematicidad y a la impropiedad de sus contenidos (así como las respectivas propuestas de reforma), en Carbonell, Miguel. La constitución pendiente. Agenda minima de reformas constitucionales $-2^{\mathrm{a}}$ edición- México: IIJ-UNAM, 2004 y en Carbonell, Miguel. Igualdad y libertad. Propuestas de renovación constitucional. México, IIJ-UNAM, CNDH, 2007. 
De hecho, dentro del capítulo dedicado a los derechos fundamentales (los que, siguiendo la tradición de hace dos siglos, todavía se les llama "garantías individuales"), se pueden encontrar cuestiones relativas a la economía nacional, a la planeación del desarrollo o incluso a los órganos constitucionales autónomos, los cuales deberían haberse previsto dentro de la parte dedicada a la división de poderes. Entre las reformas que han suscitado un mayor debate, podemos señalar una del año 2001 por medio de la cual se incluye una extensa regulación de los derechos de los pueblos y comunidades indígenas y otra de junio de 2008 para introducir una detallada regulación de las bases del procedimiento penal ${ }^{20}$.

Las constituciones más recientes (Venezuela, Ecuador y Bolivia) contemplan amplios catálogos de derechos, construidos sobre la base de los ya señalados, aunque también expresan ciertas preocupaciones locales; en su tramitación y proceso de aprobación han sido objeto de intensos debates ${ }^{21}$.

\section{DERECHOS Y CONSTITUCIONALISMO CONSERVADOR}

Cabe apuntar que no todo el contenido de las declaraciones de derechos que se encuentran en el constitucionalismo de América Latina responde a estándares progresistas y respetuosos de la dignidad humana. Hay también, infortunadamente, un "constitucionalismo conservador" en la región, que ha dejado su huella en las correspondientes secciones que recogen los derechos fundamentales ${ }^{22}$.

Aunque los ejemplos podrían multiplicarse, valga la pena ilustrar lo dicho con al menos los siguientes:

A) La Constitución de El Salvador establece, en su artículo 13 párrafo cuarto, lo siguiente: "Por razones de defensa social, podrán ser sometidos a medidas de seguridad reeducativas o de readaptación, los sujetos que por su actividad antisocial, inmoral o dañosa, revelen un estado peligroso y ofrezcan riesgos inminentes para la sociedad o para los individuos". Parece difícil reunir más ideas retrógradas en un solo párrafo. Se trata de una norma que viola varios de los más esenciales principios de cualquier sistema penal democrático y que refleja ideas del siglo XIX, por decir lo menos. Con normas como esta se autoriza al Estado a llevar a cabo casi cualquier tipo de represión

20 Sobre la reforma penal de 2008, García Ramírez, Sergio. La reforma penal constitucional (2007-2008). México: Porrúa, 2008; Carbonell, Miguel. Bases constitucionales de la reforma penal. México: IIJ-UNAM, Gobierno del Estado de Chihuahua, 2008, y Carbonell, Miguel, Los juicios orales en México. México, Porrúa, UNAM, RENACE, 2010.

21 Ver las contribuciones que, sobre tales constituciones, figuran en Carbonell, Miguel, et al. (coordinadores). Tendencias del constitucionalismo en Iberoamérica. México: IIJ-UNAM, IDEA Internacional, AECID, IIDC, 2009.

22 Las raíces históricas de este fenómeno han sido explicadas por GARGarella, Roberto, en su libro Los fundamentos legales de la desigualdad. El constitucionalismo en América (1776-1860). Madrid, España: Siglo XXI editores, 2005, pp. 85 y siguientes. 
sobre las personas, no siendo necesario ni siquiera que hayan hecho algo, puesto que se habla en ese precepto de conceptos tan evanescentes como "defensa social", "actividades antisociales, inmorales o dañosas", "estado peligroso" y "riesgos inminentes". Es decir, en ese artículo se contiene un compendio de todo aquello contra lo que lucha el derecho penal garantista ${ }^{23}$.

La misma Constitución ordena al Estado "fomentar" el matrimonio (artículo 32), y regular las relaciones familiares resultantes de la unión estable de un varón y una mujer (artículo 33). Parece obvio el sesgo conservador de tales preceptos, respecto de los "modelos" de convivencia familiar constitucionalmente aceptados. La idea de fomentar el matrimonio desde el ámbito de actuación de los poderes públicos supone implícitamente minusvalorar otras formas de convivencia, bajo un régimen jurídico no matrimonial.

La referencia a las relaciones familiares estables entre un hombre y una mujer conlleva una descalificación poco menos que explícita de las relaciones homosexuales, generando de esa forma una discriminación hacia las personas que toman la decisión de convivir con otra de su mismo sexo. Una discriminación que está inexplicablemente prevista en el texto de la norma suprema. Compárese ese anacronismo con el modelo mucho más progresista del artículo 66.9 de la Constitución de Ecuador, según el cual se reconoce y se garantiza a todas las personas "El derecho a tomar decisiones libres, informadas, voluntarias y responsables sobre su sexualidad, y su vida y orientación sexual..."24.

B) La Constitución de Guatemala deja zanjado el tema de los derechos de la mujer sobre su propio cuerpo al disponer que se protege la vida humana desde su concepción (artículo 3), con lo cual envía un mensaje muy claro al legislador para que tome medidas represivas contra las mujeres que decidan interrumpir voluntariamente su embarazo.

La misma Constitución guatemalteca dispone que se puede imponer la pena de muerte pero no a las mujeres (artículo 18), a la vez que indica que las personas presas "Deben ser tratados como seres humanos" (artículo 19) 25 .

Llama también la atención el trato preferencial que se le da a la Iglesia Católica, cuya

23 Expuesto de forma insuperada por Ferrajoli, Luigi, en Derecho y razón. Teoría del garantismo penal-9a edición- Madrid, España: Trotta, 2009.

24 Aunque cabe anotar que la misma Constitución ecuatoriana señala que el matrimonio "es la unión entre hombre y mujer" (artículo 67 párrafo segundo), con lo cual se suma a la visión conservadora de una parte del constitucionalismo latinoamericano. Para un primer acercamiento al debate sobre el matrimonio homosexual, Gerstmann, Evan. Same-sex marriage and the Constitution. Cambridge: Cambridge University Press, 2004.

25 Puede uno imaginarse la terrible realidad del sistema penitenciario guatemalteco para que haya sido necesario introducir un mandato tan básico y obvio. 
personalidad jurídica se reconoce desde el propio texto constitucional, mientras que las demás iglesias y cultos religiosos reciben un trato diferenciado (artículo 37). El trato favorable se extiende, en el mismo precepto, a las propiedades de la Iglesia Católica; las propiedades de las demás congregaciones no son siquiera mencionadas.

Los dos ejemplos anteriores, referidos a El Salvador y a Guatemala, no son los únicos; su cita quiere simplemente ilustrar sobre la existencia de un cierto modo de comprensión de los derechos fundamentales, marcado por su carácter conservador. Obviamente, las constituciones pueden ser más o menos progresistas, de acuerdo a las condiciones ideológicas y políticas del país en que se escriben y del momento en que son promulgadas. Pero no podemos pasar por alto el hecho evidente de que los derechos fundamentales deben estar al servicio de valores emancipatorios y no anclarse en realidades que mantienen vivos vínculos históricos de sujeción sobre ciertos sujetos o que reconocen primacía a algunos credos religiosos, vulnerando de esa manera la laicidad del Estado y el derecho a la no discriminación por motivos de religión ${ }^{26}$.

Respecto del derecho internacional de los derechos humanos, hemos visto en las últimas décadas el surgimiento muy fecundo de tratados internacionales en la materia, tanto universales como regionales. Dentro de la región destaca la aplicación cada vez más amplia y profunda de la Convención Americana de Derechos Humanos y de sus protocolos (sobre todo el de San Salvador, referido a los derechos económicos, sociales y culturales).

Esa preeminencia de la Convención se ha correspondido por el papel tan relevante y fructífero de la Corte Interamericana de Derechos Humanos, cuya jurisprudencia cada vez es más conocida, discutida y aplicada no solamente en el ámbito internacional, sino también en el de las jurisdicciones domésticas ${ }^{27}$.

Poco a poco, América Latina parece ir comprendiendo que la globalización no solamente significa libertad de comercio y reducción de aranceles, sino también Estado de derecho y respeto por los derechos fundamentales de todas las personas ${ }^{28}$. Las visiones reduccionistas y pueblerinas se han ido marginando a la par del surgimiento de una corriente intelectual que aboga por la internacionalización de los derechos fundamentales, con base

26 El significado de los derechos fundamentales como parámetros de emancipación y de lucha contra las discriminaciones históricamente existentes ha sido expuesto por Luigi Ferrajoli en muchas de sus obras; por ejemplo en Carbonell, Miguel (editor). Democracia y garantismo. Madrid, España: Trotta, 2008, pp. 42-59.

27 García Ramírez, Sergio (coordinador). La jurisprudencia de la Corte Interamericana de Derechos Humanos, 5 tomos. México: UNAM, 2006-2008. Ver al respecto las consideraciones de Carpizo, Jorge, "Tendencias actuales del constitucionalismo latinoamericano" en CARbonell, Miguel, et al. (coordinadores). Tendencias del constitucionalismo en Iberoamérica, op. cit. (n. 21), pp. 20-21.

28 Carbonell, Miguel y Vázquez, Rodolfo (compiladores). La globalización y el orden jurídico: reflexiones contextuales. Bogotá: Universidad Externado de Colombia, 2007. Ver, sobre todo, los ensayos de Francisco J. Laporta y Marisa Iglesias Vila, incluidos en ese volumen. 
no en posturas ideológicas, sino meramente positivistas: ese derecho internacional ya existe, es obligatorio y por tanto hay que exigir su aplicación sin fisuras ni excusas de ningún tipo.

\section{PLANO TEÓRICO}

En los últimos años la teoría constitucional referida a los derechos fundamentales también ha ido creciendo de forma significativa, si bien es cierto que todavía nos encontramos por detrás de los desarrollos que se pueden observar en algunos países europeos (sobre todo en Alemania e Italia) o incluso muy por detrás de la teoría constitucional de los Estados Unidos, que tiene entre sus temas favoritos precisamente el de los derechos.

Por esa misma razón, los desarrollos regionales se han visto alimentados (creo que de forma muy positiva) por planteamientos que vienen de otros países. En los últimos años la corriente que se ha ido implantando progresivamente es la del neoconstitucionalismo, cuyos autores más conocidos han sido analizados en muchas escuelas y facultades de derechos de América Latina, tanto a nivel de licenciatura como de posgrado. No es extraño que en nuestras aulas cientos de profesores repitan con frecuencia los nombres de Ronald Dworkin, Robert Alexy, Luigi Ferrajoli, Gustavo Zagrebelsky y el de algún neoconstitucionalista autóctono como Carlos Santiago $\mathrm{Nino}^{29}$. Las posturas de algunos de estos teóricos incluso han servido de guía para la jurisprudencia; por ejemplo, la Corte Constitucional colombiana se ha inspirado con frecuencia en la teoría de los principios de Robert Alexy o en su comprensión de la manera en que puede hacerse la ponderación de derechos; de la misma forma, la Suprema Corte de México se ha beneficiado de las aportaciones de Luigi Ferrajoli (aunque no siempre lo haya reconocido de forma expresa).

Afortunadamente, luego de unos años de intensas lecturas neoconstitucionalistas, los teóricos de América Latina están comenzando a hacer sus propios planteamientos y a aportar una visión moderna de los derechos fundamentales a la teoría jurídica local ${ }^{30}$. Los principales desarrollos se observan en Argentina, Brasil, Colombia y México, aunque se está produciendo un movimiento muy importante en el Perú y en Ecuador, países en los que veremos en los próximos ańos interesantes aportaciones teóricas.

Como sucede en otras latitudes, las bases conceptuales con las que trabajamos el tema de los derechos fundamentales los constitucionalistas, han sido suministradas por teóricos y filósofos del derecho.

Tradicionalmente hemos tomado como fuente de inspiración a la doctrina europea,

29 Un panorama sobre el movimiento del nuevo constitucionalismo puede verse en Carbonell, Miguel (editor). Neoconstitucionalismo(s). - -4a edición- Madrid, España: Trotta, 2009.

30 Algunos planteamientos de importantes teóricos de América Latina se han recogido en Carbonell, Miguel (editor). Teoría del neoconstitucionalismo. Ensayos escogidos. Madrid, España: Trotta, 2007. 
lo que resulta natural si tomamos en cuenta el alto número de estudiantes de posgrado que han pasado por las aulas de universidades españolas o italianas; esos estudiantes trajeron los referentes de sus maestros europeos, sobre todo a partir de los ańos 70 del siglo pasado.

En los últimos años, sin embargo, se observa un cambio de tendencia: hemos comenzado a mirar sin traumas históricos ni complejos de inferioridad hacia la teoría constitucional de los Estados Unidos. Las tareas de traducción, intensamente desarrolladas en Argentina, Colombia, México y, en menor medida, en Perú, han sido una puerta de entrada para que nuestros estudiantes se pudieran familiar con autores como John Rawls, Richard Posner, Ronald Dworkin, Owen Fiss, Bruce Ackerman, Duncan Kennedy, Geoffrey Stone, Sanford Levinson, Jack Balkin, Laurence Tribe, Mark Tushnet, etcétera. Desde luego, la mayor parte de las traducciones del inglés se siguen haciendo en España, donde han publicado obras de algunos de los profesores ya mencionados, pero también de Jeremy Waldron, Cass Sunstein, Will Kymlicka, etcétera.

También ha sido notable la influencia de la doctrina alemana, la cual ha aportado - de forma directa e indirecta- ideas muy importantes para el debate latinoamericano sobre los derechos fundamentales y sobre el constitucionalismo democrático en general. Los dos nombres que más se han repetido en la región creo que son los de Robert Alexy y Peter Häberle ${ }^{31}$.

La lectura directa de sus textos ha sido posible gracias a traducciones y publicaciones que han aparecido primero en Espańa, pero luego también en Brasil, Perú, Colombia, Ecuador y México. Pero, además, algunos destacados teóricos de América Latina, sobre todo de generaciones más o menos recientes, se han formado en Alemania y han escrito grandes trabajos bajo la influencia de los citados profesores. Tal es el caso, por citar solamente algunos ejemplos, de César Landa de Perú, de Rodolfo Arango, Carlos Bernal, Gloria Lopera y Alexei Julio Estrada de Colombia, de Ingo W. Sarlet de Brasil o de Laura Clérico de Argentina. Algunos de ellos son grandes promesas de la teoría jurídica latinoamericana, quienes con el tiempo deberán ocupar el lugar de los autores ya consagrados como Héctor Fix Zamudio, Carlos S. Nino, Germán Bidart, Allan Brewer Carías y otros gigantes del pensamiento de nuestra región.

31 Antes de ellos, ha sido de gran importancia el pensamiento de Konrad Hesse, que ha llegado hasta los constitucionalistas de América Latina gracias a la traducción que hizo Pedro Cruz Villalón de algunos de sus textos más conocidos, así como del mismo trabajo de traducción de su obra realizado en Brasil (país en el que incluso han traducido más materiales, en comparación con los que fueron en su momento seleccionados por Cruz Villalón). Las referencias son: Hesse, Konrad. Escritos de derechos constitucional -2a edición- Madrid, España: CEC, 1992; Hesse, Konrad. Elementos de direito constitucional da República Federal da Alemanha. Pôrto Alegre, Sergio Fabris editor, 1998. 


\section{RIESGOS Y PERSPECTIVAS}

Desde luego, en toda nuestra América Latina, los derechos fundamentales (y el régimen constitucional en su conjunto) siguen corriendo graves riesgos de retroceso, dada su precariedad. Describir tales riesgos podría llevarnos cientos de páginas, pero creo que se deben apuntar al menos los siguientes:

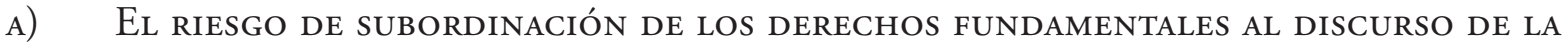 SEGURIDAD}

La crisis de seguridad pública que se vive en muchos países de la región ha influido de forma importante en la percepción social alrededor de los derechos fundamentales. Los habitantes de América Latina se preguntan ¿para qué deben servir los derechos humanos?, ¿qué tipo de castigos merecen los responsables de cometer crímenes atroces?, ¿cómo lograr que las autoridades sean eficaces en su combate contra la delincuencia organizada, sin que por alcanzar dicha eficacia terminen violando derechos?, ¿qué autoridades deben participar en la lucha contra el delito?, ¿̇requiere el Estado tomar medidas excepcionales, como la suspensión de derechos, para frenar la ola de delincuencia que afecta a una parte considerable de nuestros países?

Todas estas preguntas se intentan responder en la actualidad desde un contexto social y político marcado por el miedo. No es algo del todo nuevo, aunque quizá sí lo sea por lo que respecta a la dimensión del fenómeno dentro de la sociedad mexicana, que ha presenciado actos de una brutalidad inusitada en los tiempos recientes. La historia de la humanidad ha estado marcada por el miedo, como lo han reconocido destacados pensadores entre los que se suele citar a Hobbes. El miedo está presente en nuestras vidas desde la infancia. Ha incidido en el rumbo de algunas de nuestras decisiones desde tiempos remotos y, de alguna manera, lo sigue marcando en la actualidad.

De hecho, nunca como en nuestro tiempo ha existido una estrategia tan obvia y tan deliberada para extender el miedo entre la población y contribuir de esa manera a determinar la forma en que vivimos y las decisiones que tomamos.

El miedo de nuestra época se ha vuelto disperso, omnipresente y además es multicausal, pues lo mismo proviene de los riesgos que derivan del cambio climático que de factores vinculados con la inseguridad pública. Y por eso mismo es más efectivo, pues como señala Bauman, "El miedo es más temible cuando es difuso, disperso, poco claro; cuando flota libre, sin vínculos, sin anclas, sin hogar ni causa nítidos; cuando nos ronda sin ton ni son; cuando la amenaza que deberíamos temer puede ser entrevista en todas partes, pero resulta imposible de ver en ningún lugar concreto"32.

32 Bauman, Zygmunt. Miedo líquido. La sociedad contemporánea y sus temores. Barcelona, Espańa: Paidós, 2007, p. 10. 
El miedo determina nuestra conducta, afecta a la libertad al ejercer influencia sobre el uso que le damos a nuestra autonomía personal y, en esa medida, se proyecta sobre el ejercicio de nuestros derechos fundamentales. Las decisiones que tomamos en nuestra vida cotidiana están condicionadas por el miedo. En esa virtud, el miedo tiene que ser un componente explicativo de cualquier análisis moderno sobre el derecho constitucional y los derechos fundamentales.

La encuesta Latinobarómetro indica, en su edición correspondiente al año 2008, que la delincuencia es el principal problema de la región, seguida por el desempleo.

En América Latina están las dos ciudades más peligrosas del mundo, si tomamos en consideración el número de homicidios por cada 100.000 habitantes. Son Ciudad Juárez en México y Caracas en Venezuela, respectivamente. Ambas ciudades se ubican, en esa vergonzante clasificación, por delante de Bagdad ${ }^{33}$.

\section{B) El RETORNo DEL AUtoritarismo}

En América Latina tenemos una larga historia de regímenes autoritarios y dictatoriales. En el pasado de todos nuestros países se encuentran interrupciones (a veces recurrentes) del orden constitucional por medio de la fuerza. La presencia de las Fuerzas Armadas también ha sido una constante.

La democracia, sin embargo, parecía estarse extendiendo sin discusión desde la década de los años 80 del siglo pasado. Hoy parece que esa creencia democrática no es tan sóli$\mathrm{da}^{34}$. Nadie puede negar que tenemos en general sistemas democráticos en la región (salvo el caso concreto de Cuba, que merecería un análisis distinto, pero que indudablemente no puede ubicarse dentro de la lista de países democráticos). Pero también es cierto que nuestras democracias tienen escasa "calidad" y que los ciudadanos desconfían profundamente de sus "representantes" populares 35.

Los datos de una encuesta aplicada en toda América Latina son por demás elocuentes. Tomemos por ejemplo los que aparecen en el Latinobarómetro 2004. A partir de 1996 la satisfacción con el funcionamiento de la democracia ha sufrido fuertes retrocesos en países como Perú, Ecuador, Paraguay y Bolivia. Se ha incrementado ligeramente (entre un 8 y un 14 por ciento) en países como Panamá, Brasil, Honduras, Venezuela, Colombia y Chile.

33 Ver la nota de www.elpais.com/articulo/internacional/violencia/desangra/Caracas/elpepiint/20091006elpepiint_8/Tes

34 Carpizo, Jorge. “Tendencias actuales del constitucionalismo latinoamericano”. En: Carbonell, Miguel, et al. (coordinadores). Tendencias del constitucionalismo en Iberoamérica, op. cit. (n. 21), p. 10.

35 Sobre el tema de la calidad democrática, lo mejor que se ha publicado en y sobre México, hasta donde tengo información, es la obra colectiva coordinada por Cansino, César y Covarnubias, Israel. Por una democracia de calidad. México después de la transición. México, CEPCOM, 2007. 
Esta falta de satisfacción se demuestra muy crudamente cuando se les pregunta a los habitantes de la región si consideran que la democracia es preferible a cualquier otra forma de gobierno.

De acuerdo con la misma encuesta, los más convencidos de las bondades de la democracia viven en Costa Rica, donde el 48\% está a favor de esa forma de gobierno sobre cualquier otra. Existen altos niveles de aceptación democrática en Uruguay (45\%), Venezuela $(42 \%)^{36}$ y Chile (40\%). Por el contrario, la menor adhesión ciudadana al régimen democrático se produce en Perú (7\%), pero le siguen muy de cerca Paraguay (13\%), Ecuador (14\%), Bolivia (16\%) y México (17\%).

Pese a estos datos, tan preocupantes, lo cierto es que la mayor parte de la población de América Latina considera que un gobierno militar no podría solucionar mejor los problemas que uno democrático, aunque también en esto las cifras son muy variables. Consideran más eficaz y mejor a un gobierno democrático sobre uno militar el $85 \%$ de los encuestados en Costa Rica, el 73\% en Uruguay y el 71\% en Panamá, pero solamente el $41 \%$ en Paraguay y el $47 \%$ en Perú y Honduras. En lo que parece haber mayor consenso es en la necesidad de "aplicar más mano dura" en el país: están de acuerdo con esto el $85 \%$ de los paraguayos y el $78 \%$ de los guatemaltecos y salvadoreños. Pero solamente están de acuerdo con esa opción el 32\% de los uruguayos y el 43\% de los brasileños.

Se trata, pese a todo, de un dato inquietante, pues demuestra que hay un segmento de la población que podría sentirse atraído por una opción de "endurecimiento" del ejercicio del poder estatal, lo que podría tener consecuencias nefastas en el desarrollo democrático de alguna nación ${ }^{37}$. Sin embargo, no debemos olvidar que estos datos suelen ser muy volátiles. Así por ejemplo, la adhesión a la democracia en México era del 45\% en 1997 (año en el que el PRI pierde la mayoría absoluta en la Cámara de Diputados) y todavía del 36\% en el 2000 (cuando el PRI pierde la Presidencia de la República), pero desciende hasta un 26\% en 2001 y hasta el $17 \%$ en 2002 y $2004^{38}$.

Como quiera que sea, no dejar de resultar preocupante para el régimen de libertades y derechos el observar la involución militarista de Honduras (con golpe de Estado incluido), la persecución del pluralismo y los factores caudillistas en Venezuela, el coqueteo con la desaparición de poderes en Bolivia, la política de mano dura en Colombia, el regreso de la tortura y otras violaciones masivas de derechos en México, etcétera. Muchos de esos fenómenos

36 Aunque es probable que la presidencia de Hugo Chávez y su acción totalitaria de los últimos años haya hecho disminuir notablemente esta cifra.

37 Es probable que este endurecimiento se haya plasmado de forma amplia (y del todo autoritaria), en el derecho penal y su correlativo derecho procesal penal; ver al respecto las reflexiones de GARGARELla, Roberto. De la injusticia penal a la justicia social. Bogotá: Siglo del Hombre, 2008.

38 Se pueden encontrar estos datos, y otros más actualizados, en www.latinobarometro.org 
reflejan, de forma directa o indirecta, la persistencia de una pulsión autoritaria que parecía haber sido expulsada de la región, pero que todo parece indicar que sigue entre nosotros.

\section{C) LA PRECARIa CUltura JURÍDica}

Hay un último factor de riesgo, de entre los muchos que podrían citarse, del que vale la pena dar cuenta. Se trata de la precaria o muy débil cultura constitucional. Desde luego, no se trata de algo específico del derecho constitucional, sino que se extiende por buena parte de los sistemas jurídicos del subcontinente. La anomia generalizada parece ser un rasgo hoy en día estructural, si bien es cierto que hay en esto grandes diferencias entre los países de América Latina ${ }^{39}$.

La falta de observancia de las normas jurídicas, la justificación de conductas ilegales, la tolerancia a todo tipo de discriminaciones, el apoyo popular a conductas a veces abiertamente delictivas, la extensión del fraude fiscal y la falta de cumplimiento en general de todo tipo de deberes cívicos, no aportan en modo alguno el mejor de los escenarios para exigir un escrupuloso cumplimiento de los derechos fundamentales por parte de la autoridad ${ }^{40}$.

Aunque debería ser precisamente en este tipo de contextos en los que mayor autoexigencia pusieran las autoridades en la observancia del régimen jurídico que las vincula, aunque fuera por la idea de ponerse como ejemplo de lo que deberían hacer también todos los ciudadanos. Infortunadamente no ha sido el caso, de modo que hemos visto y seguimos viendo amplias actitudes contrarias a la Constitución realizadas, amparadas, toleradas o auspiciadas por las autoridades que deberían ser las encargadas de garantizar el cumplimiento de las normas.

39 Un análisis que se ha vuelto todo un clásico en la materia es el de Nino, Carlos. Un país al margen de la ley -3a ediciónBuenos Aires: Ariel, 2005.

40 Ver las consideraciones al respecto en García Villegas, Mauricio (director). Normas de papel. La cultura del incumplimiento de reglas. Bogotá: Siglo del Hombre, 2009. 


\section{REFERENCIAS BIBLIOGRÁFICAS}

Alexy, Robert. "La institucionalización de los derechos humanos en el Estado constitucional democrático", Derechos y libertades, número 8. Madrid, enero-junio de 2000. Teoría de los derechos fundamentales. Madrid, España: CEPC, 2002 (reimpresión).

Bauman, Zygmunt. Miedo líquido. La sociedad contemporánea y sus temores. Barcelona, España: Paidós, 2007.

Воввіо, Norberto. L’eta dei diritti. Torino, Italia: Einaudi editore, 1990.

Cansino, César y Covarrubias, Israel. Por una democracia de calidad. México después de la transición. México, CEPCOM, 2007.

Carbonell, Miguel y Vázquez, Rodolfo (compiladores). La globalización y el orden jurídico: reflexiones contextuales. Bogotá: Universidad Externado de Colombia, 2007.

_ (editor). Neoconstitucionalismo(s). -4a edición- Madrid, España: Trotta, 2009. (editor). Teoría del neoconstitucionalismo. Ensayos escogidos. Madrid, España: Trotta, Los derechos fundamentales en México. -3a edición- México: Porrúa, UNAM, CNDH, 2009.

. La constitución pendiente. Agenda mínima de reformas constitucionales. -2a ediciónMéxico: IIJ-UNAM, 2004. 2007.

Igualdad y libertad. Propuestas de renovación constitucional. México, IIJ-UNAM, CNDH, Bases constitucionales de la reforma penal. México: IIJ-UNAM, Gobierno del Estado de Chihuahua, 2008. Los juicios orales en México. México, Porrúa, UNAM, RENACE, 2010.

et al. (coordinadores). Tendencias del constitucionalismo en Iberoamérica. México: IIJUNAM, IDEA Internacional, AECID, IIDC, 2009.

(editor). Democracia y garantismo. Madrid, España: Trotta, 2008.

CARPIZO, Jorge, “Tendencias actuales del constitucionalismo latinoamericano” En: CARbonell, Miguel, et al. (coordinadores). Tendencias del constitucionalismo en Iberoamérica. México: IIJ-UNAM, IDEA Internacional, AECID, IIDC, 2009.

Dworkin, Ronald. Los derechos en serio. Barcelona, España: Planeta-Agostini, 1993.

Ferrajoli, Luigi. Derechos y garantías. La ley del más débil -4a edición- Madrid, España: Trotta, 2004.

. Derecho y razón. Teoría del garantismo penal. -9a edición- Madrid, España: Trotta, 2009.

"Más allá de la soberanía y la ciudadanía: un constitucionalismo global”. En: CARBonell, Miguel (compilador). Teoría de la Constitución. Ensayos escogidos. -4a edición- México: IIJ-UNAM, Porrúa, 2008.

García Manrique, Ricardo. "Sentido y contenido de la Declaración de 1789 y textos posteriores", Historia de los derechos fundamentales, tomo II, volumen III. Madrid, España: Dykinson, Universidad Carlos III, 2001.

García Ramírez, Sergio. La reforma penal constitucional (2007-2008). México: Porrúa, 2008

(coordinador). La jurisprudencia de la Corte Interamericana de Derechos Humanos, 5 tomos. México: UNAM, 2006-2008. 
García Villegas, Mauricio (director). Normas de papel. La cultura del incumplimiento de reglas. Bogotá: Siglo del Hombre, 2009.

Gargarella, Roberto. Los fundamentos legales de la desigualdad. El constitucionalismo en América (17761860). Madrid, España: Siglo XXI editores, 2005.

Gargarella, Roberto. De la injusticia penal a la justicia social. Bogotá: Siglo del Hombre, 2008.

Gerstmann, Evan. Same-sex marriage and the Constitution. Cambridge: Cambridge University Press, 2004.

Hesse, Konrad. Escritos de derechos constitucional -2ª edición- Madrid, España: CEC, 1992.

Elementos de direito constitucional da República Federal da Alemanha. Porto Alegre, Sergio Fabris editor, 1998.

"Significado de los derechos fundamentales" En: Benda, et al. Manual de derecho constitucional. Madrid, España: IVAP-Marcial Pons, 1996.

Jiménez Campo, Javier. Derechos fundamentales: concepto y garantías. Madrid, Espańa: Trotta, 1999.

Nino, Carlos. Un país al margen de la ley -3ª edición- Buenos Aires: Ariel, 2005.

PRIMERA, Maye. La violencia desangra Caracas <En línea> Diario electrónico Elpais.com, Disponible en World Wide Web:<www.elpais.com/articulo/internacional/violencia/desangra/Caracas/ elpepiint/20091006elpepiint_8/Tes>

Rolla, Giancarlo. "La actual problemática de los derechos fundamentales", en: Asamblea, número 3, Madrid, junio de 2000.

20.

Derechos fundamentales, Estado democrático y justicia constitucional. México: UNAM, 